\title{
Promoting physical activity in general practice: a controlled trial of written advice and information materials
}

\author{
Ben J Smith, Adrian E Bauman, Fiona C Bull, Michael L Booth, Mark F Harris
}

\begin{abstract}
Objective-To investigate the impact of a simple written prescription for physical activity given by a general practitioner and the effect of supplementing this with mailed information materials about physical activity.

Methods-A controlled trial was conducted in 27 general practices in New South Wales, Australia. Subjects were sequential routine care patients between 25 and 65 years old. Controls $(n=386)$ were recruited first, and intervention subjects two weeks later. Intervention subjects were randomised to receive a prescription only $(n=380)$ or a prescription plus a mailed booklet $(n=376)$. Self reported physical activity levels were measured by interview at baseline, 6-10 weeks, and seven to eight months.
\end{abstract}

Results-By intention to treat, the average changes in minutes of total physical activity did not differ significantly between the groups. Inactive people in the prescription plus supplementary booklet group were significantly more likely than controls to report an increase in their physical activity by at least $60 \mathrm{~min} /$ week after 6-10 weeks (odds ratio $1.58,95 \%$ confidence interval 1.06 to 2.35). No significant short term improvements in self reported activity were shown in the prescription only group. In the supplemented group, the proportion reporting an increase in physical activity to $3344 \mathrm{~kJ} /$ week at 6-10 weeks was not significant, and neither intervention group showed significant increases in any of the outcome measures at seven to eight months by intention to treat. Treatment received analysis showed greater improvements in intervention groups, especially the prescription plus booklet group, in which the odds of inactive people in this group reporting increased activity became significant at seven to eight months.

Conclusions-A prescription for physical activity from a general practitioner, supplemented by additional written materials, can lead to modest short term improvements in self reported physical activity levels among inactive patients. A prescription alone was found not to be effective.

(Br F Sports Med 2000;34:262-267)

Keywords: physical activity; family doctors; health education; patient education; intervention studies
The public health importance of physical activity as a modifiable health risk factor has been strongly endorsed in recent years. ${ }^{1}$ One reason for this has been evidence for the link between physical activity and major causes of mortality and morbidity, particularly coronary heart disease. ${ }^{2}$ Another has been population surveys that have shown a high prevalence of inactivity in western countries. ${ }^{3-5}$ These factors suggest that modest increases in physical activity in the population, particularly among people who are inactive, could produce substantial health gains.

The apparent success of general practice based interventions in addressing lifestyle risk factors such as smoking ${ }^{6}$ and excessive alcohol intake $^{7}$ has drawn attention to the potential of strategies to promote physical activity in this setting. A survey of physical activity promotion through primary care in England identified 157 schemes of this type, ${ }^{8}$ and the growth in these programmes has led to a recent review of their effectiveness. ${ }^{9}$ In England, and elsewhere, a range of strategies have been tried, including: referring patients to local exercise facilities; prescribing unstructured exercise such as walking; considering exercise as part of multiple risk factor interventions; considering it as a single risk factor; having doctors deliver interventions; using others such as practice nurses.

The small number of studies that have investigated the effect of single risk factor interventions to promote physical activity in general practice have reported some, albeit limited, positive results. The Green Prescription trial compared the impact of verbal advice from a general practitioner with a written physical activity prescription, and found that the latter approach led to greater changes in several of the physical activity measures. ${ }^{10}$ Studies that have used the transtheoretical model ${ }^{11}$ to match intervention components to the readiness of patients to be more active, such as Project PACE $^{12}$ and the recent trial conducted by Bull and Jamrozik, ${ }^{13}$ have also shown some positive outcomes. However, reviews of research on physical activity interventions in general practice have found that the evidence in favour of these is not conclusive $\mathrm{e}^{14}$ or that the evidence is only of short term benefits. ${ }^{9}{ }^{16}$ Further studies are needed, particularly those that test interventions that take account of the barriers that general practitioners face when addressing this issue in their routine practice. These include lack of time, financial constraints, and low levels of confidence in their ability to influence patient behaviour. ${ }^{17-19}$
Accepted for publication 10 March 2000 
The Active Practice project was a trial of two levels of physical activity intervention that are relatively simple and time efficient, and may present a way of overcoming some of the barriers to physical activity promotion in general practice. These were a physical activity prescription and supplementary physical activity information booklets. In order to build on previous physical activity intervention trials, this study examined the impact of each of these components by comparing the effect of the physical activity prescription with and without supplementary materials. Furthermore, the study population for this trial was routine care patients in order to test the efficacy of this intervention as a population based strategy.

\section{Methods}

STUDY DESIGN AND SAMPLE

The study was conducted as a controlled trial. Subjects were sequential routine care patients. Controls were recruited two to three weeks before the intervention group. All intervention subjects were allocated to receive a written prescription for exercise. A random sample of half of this group was sent additional booklets.

Twenty seven volunteer general practices (55 general practitioners) were recruited through three divisions of general practice in the Sydney region (32\% of those invited).

The study population was active and inactive 25 to 65 year old patients recruited sequentially by research assistants in practice waiting rooms. Patients were invited to complete a short physical activity survey in the waiting room, then two similar surveys by telephone after two and six months. Intervention subjects were informed that they may also receive some advice from their general practitioner about exercise. Patients with poor English were not considered eligible. Controls were recruited on one day in each practice, and intervention group members over a three day period in the same practices. Two practices participated in the control phase only.

Patients were later excluded for the following reasons: not supplying a telephone contact number; a contraindication to exercise; not coming to see the doctor themselves; insufficient English to complete a telephone follow up interview; reporting a health problem at follow up that prevented 30 minutes of moderate activity; being in a poor mental state at follow up-for example, intoxicated. The last two criteria only applied to the analysis of results at the follow up period at which they were apparent. If a person was excluded on these grounds at the 6-10 week follow up, a seven to eight month follow up was still attempted.

INTERVENTIONS

Between the control and intervention phases, all participating general practitioners received a 20-30 minute training session at their surgeries on the intervention procedure. The doctors did not see the baseline physical activity measurements, which were collected by the research assistants, and were encouraged to provide a prescription that they considered appropriate for each patient. Four sequential booklets were developed for the additional mailed information intervention. Following the transtheoretical model, the booklets were developed for people in the following stages: precontemplation-that is, inactive with no intention of improving; contemplation - that is, inactive but intending to improve within six months; preparation - that is, inactive but intending to improve in the next month; action and maintenance - that is, adequately active. Using data collected from baseline surveys, a random sample of half of intervention subjects was mailed a stage matched booklet about two weeks after seeing their doctor.

MEASUREMENT

Baseline data were collected by face to face interview in practice waiting rooms and follow up measures through telephone interview at 6-10 weeks and seven to eight months. In follow up surveys, interviewers were blind to the group allocation of respondents.

Physical activity participation was measured through patient recall of the frequency and duration of walking (for 10 minutes or more for any purpose) and moderate and vigorous leisure activities in the week preceding the survey. The questions were based on two week physical activity recall questions which have been found to have acceptable retest reliability. ${ }^{20}$ Total minutes of physical activity were calculated by adding the minutes of walking, moderate and vigorous physical activity reported by subjects. Three physical activity outcome measures were derived: average change in total minutes of activity; an increase in physical activity of 60 minutes or more a week compared with baseline; attaining 3344 $\mathrm{kJ} /$ week of physical activity at follow up. The first two measures were used to detect an increase in the dosage of physical activity undertaken by subjects given that incremental gains in physical activity can be beneficial. ${ }^{21}$ The third measure (3344 kJ/week) is the recommended energy expenditure level based on epidemiological research. ${ }^{21}$ The proportion of people showing improvements in these measures was examined for $(a)$ the inactive only ( $<3344 \mathrm{~kJ} /$ week at baseline), who can benefit most from increases in physical activity, (b) all patients (inactive and active), in order to explore effects for the general undifferentiated patient population.

Data about the doctors' delivery of active prescriptions and reasons why this was not given in some cases were reported by doctors on a general practitioner record form, which was completed for each patient.

\section{ANALYSIS}

Bivariate and multivariate analyses were conducted using SPSS version 6.0 for Windows. In linear and logistic regression, the covariates were: age (25-44 or 45-65); sex; language usually spoken at home (English or non-English); educational attainment (10 years or $>10$ years); total minutes of physical activity at baseline (categorised in quintiles). Both intention to treat and treatment received analyses were conducted. In intention to treat analysis, 
Table 1 Characteristics of study participants

\begin{tabular}{|c|c|c|c|c|c|c|}
\hline & \multicolumn{3}{|c|}{ All subjects } & \multicolumn{3}{|c|}{ Inactive at baseline only $(<3344 \mathrm{kf} /$ week $)$} \\
\hline & $\begin{array}{l}\text { Control } \\
n=386\end{array}$ & $\begin{array}{l}\text { Prescription } \\
n=380\end{array}$ & $\begin{array}{l}\text { Pres. E } \\
\text { booklets } \\
n=376\end{array}$ & $\begin{array}{l}\text { Control } \\
n=209\end{array}$ & $\begin{array}{l}\text { Prescription } \\
n=238\end{array}$ & $\begin{array}{l}\text { Pres. E } \\
\text { booklets } \\
n=233\end{array}$ \\
\hline Female (\%) & 60.6 & 58.4 & 62.4 & 67.0 & 63.0 & 66.4 \\
\hline Mean age (years) & 41.8 & 43.4 & 42.6 & 41.8 & $44.3^{\star}$ & 42.9 \\
\hline Education - 10 years $(\%)$ & 49.5 & 49.5 & 50.3 & 49.8 & 52.1 & 52.4 \\
\hline Language - non-English (\%) & 12.4 & $19.2^{\star}$ & 13.8 & 15.3 & 21.8 & 15.0 \\
\hline Inactive - <3344 kJ/week (\%) & 54.1 & $62.6^{\star}$ & $62.0^{\star}$ & - & - & - \\
\hline Total min of activity (median) & 145 & $95^{\star}$ & 120 & 40 & 45 & 40 \\
\hline
\end{tabular}

${ }^{\star} \mathrm{p}<0.05$ for difference from controls.

Pres., prescription.

people lost to follow up were assumed to have the same physical activity levels as at baseline. Treatment received analysis was included to examine the relative improvements in physical activity when only people known to receive interventions were included in the analysis.

\section{Results}

STUDY GROUPS

Of the eligible patients approached, 401 controls $(60.2 \%)$ and 813 intervention subjects $(56.8 \%)$ were recruited. Some patients were later excluded because of not supplying a telephone number $(\mathrm{n}=34)$, a contraindication to exercise $(n=21)$, not coming to see the doctor themselves $(\mathrm{n}=11)$, or not having sufficient English for a telephone follow up interview (n $=6$ ). Table 1 shows that the characteristics of the experimental groups were similar except that the prescription only group (group P) had a higher proportion of non-English speaking people than the control group $(p<0.05)$. In addition, the proportion of inactive people was smaller in group $\mathrm{P}$ and the prescription plus booklet group (group $\mathrm{P}+\mathrm{B}$ ) than the control group $(p<0.05)$. Similarly, the median total minutes of exercise was lower in each intervention group at baseline. Among the subjects who were inactive at baseline, the only significant difference was the higher mean age of people in group P compared with controls.

General practitioner record forms, provided for $88.5 \%$ of intervention subjects, indicated that the active prescription was given to 468 intervention patients $(61.9 \%$ of intervention group). The most common reason reported by general practitioners for not prescribing physi- cal activity was that patients were already sufficiently active. Consequently, there were higher proportions of people in the treatment received groups $\mathrm{P}(66.7 \%)$ and $\mathrm{P}+\mathrm{B}(67.1 \%)$ who were inactive than in the control groups $(\mathrm{p}<0.01)$. In addition, the mean age of inactive people in the treatment received group P (44.6 years) was higher than that of controls (41.8 years, $\mathrm{p}<0.05)$.

At $6-10$ weeks, $91.7 \%$ of all participants completed the follow up survey, whereas at seven to eight months $82.9 \%$ of participants were successfully followed up. There were no differences in the characteristics of the subjects at baseline and each follow up.

Thirty six people were excluded from the analysis of results at 6-10 weeks because of a health problem, and two because they were intoxicated at the time of interview. At seven to eight months, 41 people were excluded for health reasons. Exclusions were equally shared between the groups.

\section{CHANGES IN PHYSICAL ACTIVITY BY INTENTION}

TO TREAT

Table 2 shows that at $6-10$ weeks the average change in total minutes of physical activity among all subjects was positive in the two intervention groups but negative in the control group. The difference between the control and intervention groups on this measure was not significant. Among all subjects at 6-10 weeks, there were also more in the intervention groups who increased their physical activity by 60 min/week compared with controls, but again these differences were not significant.

Table 2 Changes in physical activity at 6-10 weeks and seven to eight months by intention to treat

\begin{tabular}{|c|c|c|c|c|c|c|c|c|c|c|}
\hline & \multicolumn{4}{|c|}{ All subjects } & \multicolumn{6}{|c|}{ Inactive at baseline only } \\
\hline & \multicolumn{2}{|c|}{ Change in total min } & \multicolumn{2}{|c|}{60 min increase } & \multicolumn{2}{|c|}{ Change in total min } & \multicolumn{2}{|c|}{60 min increase } & \multicolumn{2}{|c|}{ Increase to $3344 \mathrm{kf} /$ week } \\
\hline & $\begin{array}{l}\text { Mean } \\
\text { time } \\
\text { (min) }\end{array}$ & $\begin{array}{l}\text { Adjusted } \\
\text { (p value) }\end{array}$ & $\%$ & $\begin{array}{l}\text { Odds ratio* } \\
(95 \% \text { CI })\end{array}$ & $\begin{array}{l}\text { Mean } \\
\text { time } \\
\text { (min) }\end{array}$ & $\begin{array}{l}\text { Adjusted } \\
\text { (p value) }\end{array}$ & $\%$ & $\begin{array}{l}\text { Odds ratio* } \\
(95 \% \text { CI })\end{array}$ & $\%$ & $\begin{array}{l}\text { Odds ratio }{ }^{*} \\
(95 \% \text { CI) }\end{array}$ \\
\hline \multicolumn{11}{|l|}{$6-10$ weeks } \\
\hline Control (n=377) & -5.7 & & 29.1 & & 62.5 & & 35.0 & & 27.0 & \multirow{3}{*}{$\begin{array}{l}1.00 \\
(0.63-1.58) \\
1.19 \\
(0.77-1.87)\end{array}$} \\
\hline Prescription $(n=364)$ & 16.3 & $\begin{array}{l}15.6 \\
(0.27)\end{array}$ & 34.2 & $\begin{array}{l}1.26 \\
(0.92-1.75)\end{array}$ & 78.0 & $19.0(0.18)$ & 40.7 & $\begin{array}{l}1.34 \\
(0.90-2.00)\end{array}$ & 25.7 & \\
\hline Prescription + booklet $(n=363)$ & 16.9 & $\begin{array}{l}15.3 \\
(0.27)\end{array}$ & 35.7 & $\begin{array}{l}1.32 \\
(0.96-1.82)\end{array}$ & 81.1 & $18.1(0.20)$ & 45.7 & $\begin{array}{l}1.58 \\
(1.06-2.35)\end{array}$ & 30.8 & \\
\hline \multicolumn{11}{|l|}{$7-8$ months } \\
\hline Control (n=373) & -22.4 & & 23.1 & & 41.2 & & 27.4 & & 16.9 & \\
\hline Prescription $(\mathrm{n}=365)$ & -7.5 & $\begin{array}{c}4.1 \\
(0.76)\end{array}$ & 24.5 & $\begin{array}{l}1.06 \\
(0.74-1.51)\end{array}$ & 60.1 & $18.2(0.19)$ & 31.6 & $\begin{array}{l}1.22 \\
(0.79-1.87)\end{array}$ & 21.5 & $\begin{array}{l}1.43 \\
(0.87-2.36)\end{array}$ \\
\hline Prescription + booklet $(n=363)$ & -3.4 & $\begin{array}{l}10.3 \\
(0.45)\end{array}$ & 27.9 & $\begin{array}{l}1.26 \\
(0.89-1.79)\end{array}$ & 65.1 & $23.6(0.09)$ & 35.7 & $\begin{array}{l}1.50 \\
(0.99-2.29)\end{array}$ & 24.4 & $\begin{array}{l}1.60 \\
(0.98-2.60)\end{array}$ \\
\hline
\end{tabular}

^Adjusted means for sex, age, educational attainment, language spoken at home, and baseline level of physical activity. 
Table 3 Changes in physical activity at 6-10 weeks and seven to eight months by treatment received

\begin{tabular}{|c|c|c|c|c|c|c|c|c|c|c|}
\hline & \multicolumn{4}{|c|}{ All subjects } & \multicolumn{6}{|c|}{ Inactive at baseline only } \\
\hline & \multicolumn{2}{|c|}{ Change in total min } & \multicolumn{2}{|c|}{60 min increase } & \multicolumn{2}{|c|}{ Change in total min } & \multicolumn{2}{|c|}{60 min increase } & \multicolumn{2}{|c|}{ Increase to $3344 \mathrm{kf} /$ week } \\
\hline & $\begin{array}{l}\text { Mean } \\
\text { time } \\
\text { (min) }\end{array}$ & $\begin{array}{l}\text { Adjusted } \\
\text { (p value) }\end{array}$ & $\%$ & $\begin{array}{l}\text { Odds ratio* } \\
(95 \% \mathrm{CI})\end{array}$ & $\begin{array}{l}\text { Mean } \\
\text { time } \\
\text { (min) }\end{array}$ & $\begin{array}{l}\text { Adjusted } \\
\text { (p value) }\end{array}$ & $\%$ & $\begin{array}{l}\text { Odds ratio* } \\
(95 \% \mathrm{CI})\end{array}$ & $\%$ & $\begin{array}{l}\text { Odds ratio } \\
(95 \% \mathrm{CI})\end{array}$ \\
\hline \multicolumn{11}{|l|}{$6-10$ weeks } \\
\hline Control $(\mathrm{n}=347)$ & -6.2 & & 31.6 & & 69.1 & & 38.7 & & 29.8 & \\
\hline Prescription $(n=205)$ & 20.8 & $9.7(0.58)$ & 39.5 & $\begin{array}{l}1.33 \\
(0.91-1.93)\end{array}$ & 95.1 & $\begin{array}{l}29.9 \\
(0.08)\end{array}$ & 49.6 & $\begin{array}{l}1.59 \\
(1.00-2.52)\end{array}$ & 30.4 & $\begin{array}{l}1.09 \\
(0.65-1.82)\end{array}$ \\
\hline $\begin{array}{l}\text { Prescription + booklet } \\
(\mathrm{n}=207)\end{array}$ & 42.8 & $12.8(0.46)$ & 43.8 & $\begin{array}{l}1.50 \\
(1.04-2.17)\end{array}$ & 86.0 & $\begin{array}{l}17.5 \\
(0.29)\end{array}$ & 50.3 & $\begin{array}{l}1.63 \\
(1.04-2.55)\end{array}$ & 31.3 & $\begin{array}{l}1.07 \\
(0.65-1.77)\end{array}$ \\
\hline \multicolumn{11}{|l|}{$7-8$ months } \\
\hline Control $(n=310)$ & -26.9 & & 27.7 & & 51.4 & & 34.2 & & 21.1 & \\
\hline Prescription $(\mathrm{n}=187)$ & -7.8 & $-9.1(0.62)$ & 31.7 & $\begin{array}{l}1.09 \\
(0.71-1.67)\end{array}$ & 67.3 & $\begin{array}{l}11.9 \\
(0.52)\end{array}$ & 39.5 & $\begin{array}{l}1.19 \\
(0.71-1.97)\end{array}$ & 22.6 & $\begin{array}{l}1.11 \\
(0.62-2.00)\end{array}$ \\
\hline $\begin{array}{l}\text { Prescription }+ \text { booklet } \\
(\mathrm{n}=183)\end{array}$ & 12.3 & $6.0(0.74)$ & 37.8 & $\begin{array}{l}1.39 \\
(0.92-2.10)\end{array}$ & 95.6 & $\begin{array}{l}43.4 \\
(0.02)\end{array}$ & 47.9 & $\begin{array}{l}1.78 \\
(1.08-2.94)\end{array}$ & 32.8 & $\begin{array}{l}1.79 \\
(1.03-3.10)\end{array}$ \\
\hline
\end{tabular}

^Adjusted means for sex, age, educational attainment, language spoken at home, and baseline level of physical activity.

Examining the inactive only at $6-10$ weeks (table 2), groups $\mathrm{P}$ and $\mathrm{P}+\mathrm{B}$ showed more change in total minutes than the control group, but the difference was small and not significant. The adjusted odds ratio for the proportion of people increasing their physical activity by $60 \mathrm{~min} /$ week showed that group $\mathrm{P}+\mathrm{B}$ improved significantly more than the control group. Group P showed a greater, but nonsignificant, change with respect to this variable than controls. There was little difference in the proportions of inactive people in any of the groups who improved their physical activity to the $3344 \mathrm{~kJ} /$ week level at the $6-10$ week follow up.

The amount of improvement in the physical activity variables was smaller at the seven to eight month follow up than at 6-10 weeks. Considering all subjects, there was an average decline in total minutes of physical activity at seven to eight months in all experimental groups. The adjusted change in total minutes of activity was similar in the intervention and control groups. Moreover, there were only small differences between the proportions of the whole groups who showed a 60 minute a week improvement in activity.

Among the inactive only at seven to eight months, group $\mathrm{P}+\mathrm{B}$ showed the greatest average improvement in total activity, followed by group $\mathrm{P}$, then controls. Neither intervention group showed significant improvements compared with controls. The pattern of effects between the groups was similar for the $60 \mathrm{~min} /$ week and $3344 \mathrm{~kJ} /$ week variables, although the odds of group $\mathrm{P}+\mathrm{B}$ improving on these measures were not significant.

CHANGES IN PHYSICAL ACTIVITY BY TREATMENT RECEIVED

In treatment received analysis, there were generally larger improvements in the intervention groups compared with the controls than was apparent by intention to treat, although the pattern of effects was similar (table 3). At 6-10 weeks, there were greater but non-significant improvements in average total minutes of activity in group $\mathrm{P}$ and group $\mathrm{P}+\mathrm{B}$ than controls, both when the whole groups and just the inactive were considered. In contrast with the intention to treat findings, whole group analysis at $6-10$ weeks showed group $\mathrm{P}+\mathrm{B}$ to have a significantly greater likelihood of increasing their physical activity by $60 \mathrm{~min} /$ week compared with controls. Also, among the inactives, group $\mathrm{P}+\mathrm{B}$ showed a significant likelihood of improvement by $60 \mathrm{~min} /$ week, whereas the improvement in group $\mathrm{P}$ on this measure was not significant.

Under treatment received conditions, the changes among the inactive at seven to eight months showed the greatest difference from the findings by intention to treat. This was apparent in the adjusted average change in total minutes among the inactive in group $\mathrm{P}+\mathrm{B}$, which reached significance. In addition, the proportions of inactive people in this group who improved by $60 \mathrm{~min} /$ week and to the 3344 $\mathrm{kJ} /$ week level were significantly greater than controls in this type of analysis.

\section{Discussion}

The active prescription, when supplemented by a stage matched information booklet, was associated with modest short term improvements in self reported physical activity among inactive general practice patients. The prescription alone did not lead to significant improvements in reported physical activity in this sample who were recruited systematically and independently of the general practitioners. There was a notable decline in reported activity between the 6-10 week and seven to eight month follow ups in all groups, and only limited evidence that the prescription plus booklet intervention had any medium term benefit.

The average change in total minutes of physical activity variable showed little difference, in most cases less than 20 minutes, between the control and intervention groups. The only significant difference observed in this measure was in the treatment received analysis, where the adjusted change in total minutes among inactive people in group $\mathrm{P}+\mathrm{B}$ was a little over 40 minutes greater than controls. This finding indicates that the brief physical activity interventions tested here do not lead to significant changes in reported activity among all people who receive these.

Using the categorical outcome measures, by intention to treat we observed that inactive 
patients in the prescription plus booklet group were significantly more likely to report a 60 $\mathrm{min} /$ week improvement in physical activity at 6-10 weeks than controls. When the whole groups were considered (including the active) there were no significant differences in the proportions who showed this amount of improvement. Thus an approach that targets the inactive may lead to greater changes in physical activity. Although this improvement was encouraging, it should be noted that inactive subjects in this group were not more likely to increase their physical activity to the 3344 $\mathrm{kJ} /$ week level after 6-10 weeks. This suggests that, although potentially effective, our prescription plus booklet intervention was not an adequate strategy to achieve public health targets for physical activity, with limited impact in the medium term.

Notable in the categorical outcome measures were the substantial proportions of the control group (mostly more than 25\%) who reported at least moderate initial increases in their physical activity. Secular trends, possibly related to seasonality, may explain some of this change. A Hawthorne effect may also have played a part in this.

The results obtained using the treatment received analysis provided corroboration of the main findings by intention to treat. That is, they indicated that the prescription plus booklet intervention had more impact than prescription alone, and by showing greater effects among treatment recipients, further suggested that it was the interventions themselves that may be influencing patients.

Central to considering the implications of these findings are the methodological limitations of this study and the effect sizes that were associated with the interventions. The use of quasi-experimental rather than random controls was a weakness in the design of the study. Drawing controls from the same practices as intervention group members, but at a slightly earlier time, was a pragmatic choice that was expected to provide similarity between the groups and yet reduce the possibility of contamination of controls. However, there were significant differences in the baseline levels of physical activity between the control and intervention groups. Although adjustment for the baseline characteristics of the groups was included in the analysis, this cannot deal with any other unknown differences between the groups that may have affected their changes in activity. Another weakness of this study was the reliance on self report outcome measures of physical activity. It is seldom feasible in real world primary care settings to use more costly objective measures of movement and energy expenditure, but it is assumed that any reporting bias by subjects was non-differential between the experimental groups, suggesting that any observed differences between groups are likely to be real.

Although these weaknesses reduce the quality of evidence that was gained, they represent common challenges in this field of research. Quasi-experimental studies are common in primary care settings, because of the risks involved in relying on busy general practitioners to implement randomisation protocols around behavioural counselling. One alternative is the use of cluster randomisation designs, randomly allocating whole practices to experimental conditions for instance, but this requires large sample sizes to accommodate clustering effects within groups, particularly when there are more than two experimental groups. The limitations of self report physical activity measures is another challenge. Objective validation of self reported activity on subsamples of study members is one way of checking the accuracy of reporting, but this is problematic when subjects are being followed up on two or more occasions, as the validation procedure itself may influence exercise behaviour. Although a number of self reported physical activity measurement tools have been independently validated, when these are applied in intervention, as opposed to prevalence, studies, there will remain the risk that a range of factors - for example, Hawthorne effects-may influence subject responses.

The other important question to consider is whether the effect sizes associated with the prescription plus stage matched booklet intervention should be considered as support for this type of brief intervention in general practice. In this regard, it is helpful to consider these findings in the light of the results of other physical activity trials in general practice. In Project PACE, doctors gave patients three to five minutes of counselling, which was written down and later reinforced by a 10 minute telephone call from a health educator. Although PACE used a more complex doctor counselling protocol, and the reinforcement measure was more intensive than our mailed booklet approach, it too only showed modest improvements among intervention subjects in the short term. In the trial of verbal advice supplemented by information booklets conducted by Bull and Jamrozik, ${ }^{13}$ there was a short term increase in number of sessions of activity among sedentary subjects who received the intervention materials, compared with controls, but no difference in total reported time spent in physical activity. The Green Prescription trial ${ }^{10}$ found that a general practitioner delivered physical activity prescription had significantly more effect in increasing the proportion of subjects who do any recreational activity at all at six weeks compared with verbal advice alone. This study too did not find significant differences in the duration of physical activity between the experimental groups, even though subjects were inactive patients who had been selected by general practitioners because they could benefit from more exercise.

Although comparison of the Active Practice project with other recent trials is difficult because of differences in the study groups, interventions, and outcome measures, the trend that emerges from these trials is that brief interventions to promote physical activity in general practice are mostly of short term benefit only. The definitive approach to promoting physical activity in general practice has not yet been found. Given that, in this study, 
the group that received the supplementary booklets showed the most improvement, it appears that subsequent reinforcement of general practitioner interventions is critical to increasing their impact. Thus even further reinforcement measures beyond a single mailed booklet - for example, through additional mailed material or active follow up by general practitioners, could strengthen the medium and even longer term effects of these interventions. These suggestions, however, remain to be empirically tested.

A continuing challenge in the development of physical activity interventions in general practice will be attempting to generalise the intervention effects that are gained from studies conducted with interested volunteer general practitioners. In this study, $32 \%$ of those general practitioners invited were willing to take part, and characteristics of nonparticipating general practitioners were not sought. However, even if the physical activity interventions, like the one tested here, only receive a modest uptake by general practitioners, this could still be an important population strategy. Developing methods to reinforce general practitioner advice on physical activity is a future challenge, and might include using information technology to supplement and extend practitioner advice.

In summary, this study has shown that the delivery of a written prescription for physical activity by general practitioners can have some impact on the reported physical activity participation of inactive patients when it is supplemented by mailed information materials. This study, and other similar trials have found only limited evidence of effects beyond the short term (6-10 weeks) and for increases to recommended levels of physical activity (3344 kJ/week). Until convincing evidence is provided otherwise, it appears that interventions focusing on brief advice have only a modest role to play in long term efforts to improve population levels of physical activity.

This study was funded by a physical activity demonstration This study was funded by a physical activity demonstration
grant (DP/96/2) from the NSW state Health Department. We grant (DP/96/2) from the NSW state Health Department. We
would like to thank the Liverpool, Western Sydney, and would like to thank the Liverpool, Western Sydney, and
Illawarra Divisions of General Practice, doctors who participated in the study, and the NSW Division of the National Heart pated in the study, and the NSW Division of the National Heart
Foundation. Frank Wallner and Alison Marshall contributed to Foundation. Frank Wallner and Alison $M$

the development of intervention materials.
Contributors: A E B was the PI on the grant, designed the Contributors: A E B was the PI on the grant, designed the
study, wrote the application, and had overall responsibility for managing the research. M L B and F C B were co-investigators, and contributed on a frequent basis to design and field work in an advisory role. B J S was the project manager, recruited doctors and patients, supervised all field work, analysed the data, and wrote the initial draft. All authors had extensive input in the writing of subsequent drafts. A E B acts as guarantor for the paper.

1 US Department of Health and Human Services. Physical activity and health: a report of the US Surgeon General. Atlanta, GA: US Department of Health and Human Services, Centers for Disease Control and Prevention, National Center for Chronic Disease Prevention and Health Promotion, 1996.

2 Berlin JA, Colditz GA. A meta-analysis of physical activity in the prevention of coronary heart disease. Am $\mathcal{F}$ Epidemiol 1990;132:612-28.

3 Centers for Disease Control and Prevention. Behavioural risk factor surveillance system survey. Atlanta, GA: Division of Adult and Community Health, National Center for Chronic Disease Prevention and Health Promotion, 1994.

4 Activity and Health Research. Allied Dunbar national fitness survey. London: The Sports Council and Health Education survey. London: Th.

5 Australian Bureau of Statistics. National health survey. Summary results: Australian states and territories. Canberra: Australian Bureau of Statistics, 1997, Catalogue no 4368.0

6 Kottke TE, Battista RN, DeFriese GH, et al. Attributes of successful smoking cessation interventions in medical practice: a meta-analysis of 39 controlled trials. FAMA 1988;259:2883-9.

7 Bien TH, Miller WR, Tonigan JS. Brief interventions for alcohol problems: a review. Addiction 1993;88:315-36.

8 Fox K, Biddle S, Edmunds L, et al. Physical activity promotion through primary care in England. Br f Gen Pract 1997; 47:367-9.

9 Riddoch C, Puig-Ribera A, Cooper A. Effectiveness of physical activity promotion schemes in primary care: a review. condon: Health Education Authority, 1998.

10 Swinburn BA, Walter LG, Arroll B, et al. The Green Prescription study: a randomised controlled trial of written exercise advice provided by general practitioners. $A m$ F Public Health 1998;88:288-91.

11 Prochaska JO, DiClemente CC. Stages and processes of self-change of smoking: toward an integrative model of change. F Consult Clin Psychol 1983;51:390-5.

12 Calfas KJ, Long BJ, Sallis JF, et al. A controlled trial of physician counselling to promote the adoption of physical activity. Prev Med 1996;25:225-33.

13 Bull FC, Jamrozik K. Advice from a family physician can help sedentary patients to become active. Am $\mathcal{F}$ Prev Med 1998;15:85-94

14 Ashenden R, Silagy C, Weller D. A systematic review of the effectiveness of promoting lifestyle change in general praceffectiveness of promoting lifestyl
tice. Fam Pract 1997;14:160-75.

15 Eaton CB, Menard LM. A systematic review of physical activity promotion in primary care office settings. $\mathrm{Br} \mathcal{F}$ activity promotion in primar

16 Simons-Morton DG, Calfas KJ, Oldenburg B, et al. Effects of interventions in health care settings on physical activity or cardiorespiratory fitness. Am F Prev Med 1998;15:41330

17 Williford HN, Barfield B, Lazenby RB, et al. A survey of physicians' attitudes and practices related to exercise promotion. Prev Med 1992;21:630-6.

18 Orleans CT, George LK, Houpt JL, et al. Health promotion in primary care: a survey of US family practitioners. Prev Med 1985;14:636-47.

19 Bull FCL, Schipper ECC, Jamrozik K, et al. Beliefs and behaviour of general practitioners regarding promotion of
physical activity. Aust f Public Health 1995;19:300-4.

20 Booth ML, Owen N, Bauman AE, et al. Retest reliability of recall measures of leisure-time physical activity in Australian adults. Int 7 Epidemiol 1996;25:153-9.

21 Pate RR, Pratt M, Blair SN, et al. Physical activity and public health: a recommendation from the Centers for Disease Control and Prevention and the American College of Sports Medicine. FAMA 1995;273:402-7.

Take home message

Although primary care remains an appealing setting for physical activity interventions, the evidence on the effectiveness of this setting is not established. In this Australian trial, improvements in physical activity levels of patients were only achieved when doctor advice and exercise prescription were supplemented by mailed materials, and even then, effects were only short term. 